\title{
Pilot study of photoallergic
}

reactions to non-steroidal anti-

inflammatory agents,

chemicals used in dental

composites and antiseptic

agents among dental

professionals

\author{
Maya Lyapina ${ }^{1}$, Maria Dencheva ${ }^{2}$, Assya Krasteva ${ }^{2}$, \\ Mariana Tzekovaa ${ }^{2}$, Mariela Deliverska ${ }^{3}$, Angelina \\ Kisselova $^{2}$, Stella Christova ${ }^{2}$, Jordan Galabov ${ }^{2}$.
}

1. Medical University, Sofia, Bulgaria, Medical Faculty, Department "Hygiene, Medical Ecology and Nutrition";

2. Medical University, Sofia, Bulgaria, Faculty of Dental Medicine, Department "Oral and Image Diagnostics";

3. Military Medical Academy, Head of Legal Department, Sofia, Bulgaria;

\section{Abstract}

The purpose of the present pilot study was to evaluate the incidence of photoallergic reactions to selected non-steroidal anti-inflammatory agents, chemicals used in dental composites and antiseptic agents (Triclosan) among dental professionals (dentists, nurses and attendants) and dental patients. A total of 59 participants were included: 25 occupationally exposed dental 
professionals and 34 dental patients. Skin photopatch testing with Methylhydroquinone, Camphorquinone, Benzydamine hydrochloride, Triclosan, and Ibuprofen (Chemotechnique Diagnostics) was performed, according to: Photopatch testing: a consensus methodology for Europe. No reactions of photosensitization to Methylhydroquinone and Camphorquinone were observed. Highest rates of contact sensitization to Triclosan, especially among men and dental patients were established, but no reactions of photosensitization. Our results indicate risk of sensitization to Triclosan in consumers, but not in occupational exposure. We could consider lbuprofen as second of importance allergen for all the groups defined by us, but no photosensitizing action was found. The only positive skin patch test reactions after UV-irradiation observed in the present study were to Benzydamine hydrochloride, in the groups of women $(0.0 \%$ vs $6.9 \%)$ and in the one of dental professionals (0.0\% vs $4.0 \%)$. According to our results, Benzydamine hydrochloride is the only of the substances exerting photosensitizing action, and dental professionals and women could be outlined as groups at risk. However, further work is needed to validate the reliability of these findings.

Keywords: photosensitization, dental professionals, photopatch skin testing, Methylhydroquinone, Camphorquinone, Benzydamine hydrochloride, Triclosan, Ibuprofen

\section{Introduction}

Dental materials and products are widely used, and some of their ingredients may cause problems both for dental personnel because of occupational exposure and for patients undergoing dental treatment. Photoallergic contact dermatitis is a variant of allergic contact dermatitis. In the photoallergic variant, subsequent to the exposure to exogenous hapten irradiation, typically with ultraviolet (UV) light, is required to initiate the pathologic processes. The photons deliver energy for either creating covalent bonds between hapten and endogenous protein (formation of antigenic photo adducts), or converting a prohapten into the actual sensitizing hapten $(1,2)$.

Photosensitization reactions are a continuously growing area of research which deals with the desirable and undesirable processes induced in biological systems by the absorption of UV. The common photosensitizing agents include chemicals used in dental composites, antiseptic agents, and non-steroidal anti-inflammatory agents widely used in dental practice. Photopatch testing is an effective approach for the diagnosis of photodermatitis or unclear photoreactions and helps in determining the sensitizing potentials of commonly used agents $(3,4)$. Quite a few studies are available in regard to the incidence of reactions of photosensitization among dental professionals.

\section{Aim}

The purpose of the present pilot study was to evaluate the incidence of photoallergic reactions to selected non-steroidal anti-inflammatory agents, chemicals used in dental composites and antiseptic agents (Triclosan) among dental professionals (dentists, nurses and attendants) and dental patients.

\section{Materials and methods}


A total of 59 participants, divided into two groups, were included in the study: occupationally exposed dental professionals, and randomly chosen dental patients of different gender, age and occupations, the main inclusion criteria being the lack of occupational exposure to dental materials. Data regarding age and gender characteristics are presented in Table 1.

Table 1. General characteristics of the studied groups of individuals

\begin{tabular}{|l|c|c|c|c|}
\hline \multirow{2}{*}{ Studied groups } & \multirow{2}{*}{$\begin{array}{c}\text { Age } \\
\text { (years) } \\
(\mathbf{M} \pm \text { SD) }\end{array}$} & $\begin{array}{c}\text { Women } \\
\mathbf{n}(\%)\end{array}$ & $\begin{array}{c}\text { Men } \\
\mathbf{n}(\%)\end{array}$ & \\
\cline { 3 - 5 } & & $14(79,5)$ & $20(20.5)$ & $\mathbf{3 4}$ \\
\hline Dental patients & $40.41 \pm 14.17$ & $15(72.3)$ & $10(27.7)$ & $\mathbf{2 5}$ \\
\hline Dental professionals & $43.57 \pm 8.76$ & $\mathbf{2 9}(\mathbf{4 9 . 2 )}$ & $\mathbf{3 0 ( 5 0 . 8 )}$ & $\mathbf{5 9}$ \\
\hline \multicolumn{1}{|c|}{ Total } & $42.23 \pm 16.3$ & \multicolumn{2}{|c|}{} \\
\hline
\end{tabular}

The study was granted by the Medical University - Sofia, Grant № 48/2014, and was approved by the Medical Ethics Board at the Medical University of Sofia. All participants were informed about the purpose of the study and gave their written informed consent.

\section{Skin photopatch testing}

Skin photopatch testing with the following photoallergens, included in the European Photopatch Extended

( E-1000) and Dental Screening (DS-1000 - Chemotechnique Diagnostics) Series - Methylhydroquinone (1.0\% pet), Camphorquinone (Bornanedione - $1.0 \%$ pet), Benzydamine hydrochloride $(2.0 \%$ pet), Triclosan (2.0\% pet), and lbuprofen (5.0\% pet) was performed, according to Photopatch testing: a consensus methodology for Europe (5), by application of the allergens in IQ-Ultra hypoallergenic patches of Chemotechnique Diagnostics (IQ Chambers $\AA$, Vellinge, Sweden) on the mid upper back skin, avoiding 3 to $5 \mathrm{~cm}$ on either side of the vertebrae. Duplicate sets on left and right side of the back of the patient were applied for $48 \mathrm{~h}$, after which both sets were removed. At this point, one set should be covered with an ultraviolet (UV) opaque material and the other irradiated with a calibrated metered broad-spectrum UVA source (UV-Therapy and Photodiagnosis system UV 236 PUVA) with a dose of $5 \mathrm{~J} / \mathrm{cm} 2$. Readings were recorded using the International Contact Dermatitis Research Group (ICDRG) scoring system with readings before irradiation, immediately after irradiation and also 48 and/or $72 \mathrm{~h}$ thereafter. An interpretation key based on recommendations by the ICDRG was applied (Table 2). 
Table 2. Interpretation key of skin patch test results based on International Contact Dermatitis Research Group

\begin{tabular}{|l|c|}
\hline \multicolumn{1}{|c|}{ Symbol } & Meaning \\
\hline$(-)$ & negative reaction \\
\hline $\boldsymbol{P}$ & doubtful reaction \\
\hline $\boldsymbol{+}$ & weak positive reaction (non-vesicular) \\
\hline $\mathbf{+ +}$ & $\begin{array}{c}\text { strong positive reaction (oedematous or } \\
\text { vesicular) }\end{array}$ \\
\hline $\mathbf{+ + +}$ & extreme positive reaction (ulcerative or \\
& bullous) \\
\hline $\mathbf{I R}$ & irritant reaction \\
\hline
\end{tabular}

Lack of anti-allergic medication constituted a mandatory condition before placing the patches and during the testing.

\section{Statistical methods}

The statistics were calculated with SPSS 19.0. Available tools for cross-tabulation statistics were used Fisher Exact Test for statistical significance. Values of $\mathrm{P}<0.05$ were accepted as statistically significant.

\section{Results}

Distribution by gender was uniform. The mean duration of occupational exposure of dental professionals to photosensitizers as ingredients of dental materials is 18 years, and $68 \%$ of them were exposed up to 20 years.

Data concerning the incidence of positive skin patch test reactions to the tested substances, before and after UV-irradiation (distribution by gender and groups defined by occupational exposure in dentistry) are presented in Table 3. 
Table 3. Distribution of positive skin patch test reactions to tested substances, before and after UV-irradiation among the studied groups.

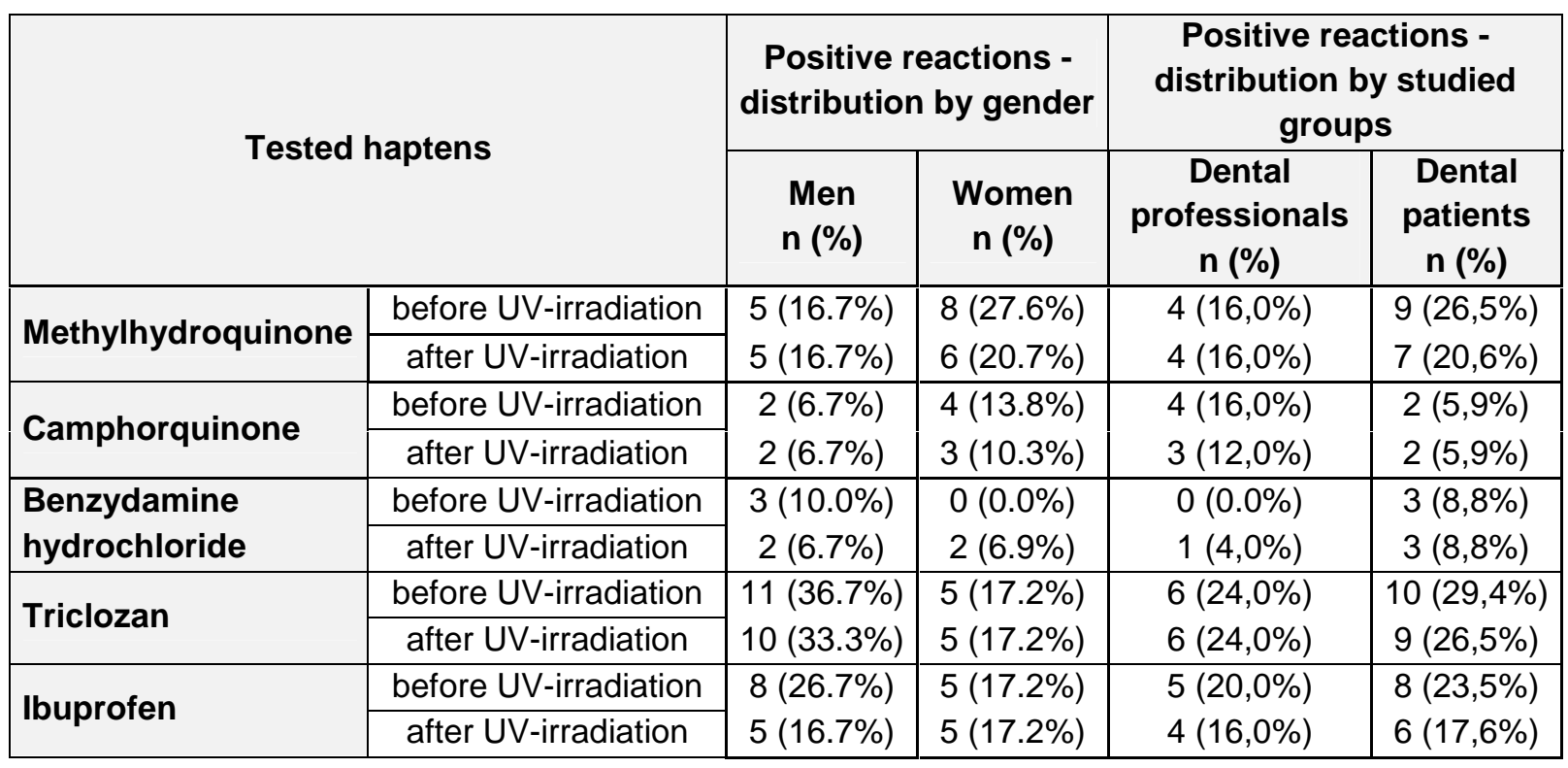

No reactions of photosensitization to Methylhydroquinone and Camphorquinone were observed in our study. The incidence of positive reactions to Methylhydroquinone after UV-irradiation decreased in the group of women ( $27.6 \%$ before vs $20.7 \%$ after UV-irradiation) and the group of dental patients ( $26.5 \%$ vs $20.6 \%)$, without statistical significance $(p=0.48, p=0.46)$.

Concerning the incidence of positive reactions to Camphorquinone after UV-irradiation, it was lower among women (13.8\% vs $10.3 \%$ ) and dental professionals (16.0\% vs $12.0 \%)$. No statistically significant differences were observed $(p=0.63, p=0.54)$.

The only positive skin patch test reactions after UV-irradiation observed in the present study were to Benzydamine hydrochloride, in the group of women (0.0\% vs $6.9 \%)$ and in the one of dental professionals $(0.0 \%$ vs $4.0 \%)$; nevertheless, a decrease of the incidence was observed among the group of men ( $10.0 \%$ vs $6.7 \%)$. No statistical significance was revealed $(p=0.68, p=0.63)$.

The results from photopatch testing to Triclozan are available in table 3 . The sensitization rates to this substance were higher when compared with the others studied among men and dental patients. No positive photopatch test reactions were observed in any of the defined groups. Furthermore, a decreased incidence of positive skin patch test reactions after UV-irradiation was observed in the groups of men ( $36.7 \%$ vs $33.3 \%$ ) and the one of dental patients (29.4\% vs $26.5 \%$ ). Nevertheless, no statistical significance was revealed $(p=0.23, p=0.54)$.

Finally, due to the wide use of Ibuprofen as non-steroidal anti-inflammatory drug, in the present pilot study we tested its possible photosensitizing action. No reactions of photosensitization were manifested in any of the defined groups of individuals; a decreased incidence of positive skin patch test reactions after UV- 
irradiation was observed in the groups of men (26.7\% vs $16.7 \%)$, of dental professionals $(20.0 \%$ vs $16.0 \%)$ and dental patients (23.5\% vs $17.6 \%)$. Statistical significance was no established $(p=0.61, p=.58)$.

\section{Discussion}

Methylhydroquinone is used as a stabilizer and antioxidant in acrylic monomers to prevent polymerization and as an antioxidant for fatty esters, linseed oil and other inedible fats and oils. It is also used as an intermediate to manufacture other stabilizers, dyes, pharmaceuticals and plasticizers (6). Positive patch test reactions to methylhydroquinone in relation to clinical presentation in dental personnel were observed (7).

Our results don't confirm the hypothesis about possible manifestation of photosensitization reactions to Methylhydroquinone. Nevertheless, the relative high incidence of positive skin patch test reactions to this substance in the group of women - $27.6 \%$, decreasing to $20.7 \%$ after UV-irradiation should be emphasized.. Statistical significance was not found, but it could be speculated that women appear to be a more vulnerable group for sensitization.

Camphorquinone (1,7,7 - trimethylbicyclo [2.2.1] heptane - 2,3 - dione, CQ) belongs to the aliphatic $\alpha$ diketones and is by far the most widely used photoinitiator for visible-light photocrosslinking in methacrylate-based dental restorative polymers and other biomedical applications (8-10). CQ absorbs light in the UV-region at 200-300 nm and in the visible light region at $467 \mathrm{~nm}$ (responsible for its yellow color) (11). The concentration of $C Q$ in dental resins varies significantly. An analysis of seven visible-lightcurable dental composite resins revealed that the concentration of $C Q$ in the resin phase was between 0.17 and $1.03 \mathrm{wt} \%(12,13)$.

No reactions of photosensitization to Camphorquinone were observed as well. Though without statistical significances, a decreased incidence of positive skin patch test reactions after UV-irradiation among women and dental professionals was observed. No risk of photosensitization to CQ could be suggested.

Benzydamine (also known as Tantum Verde), available as the hydrochloride, is a locally-acting nonsteroidal anti-inflammatory drug with local anaesthetic and analgesic properties for pain relief and antiinflammatory treatment of inflammatory conditions of the mouth and throat. It acts by inhibiting the body's production of prostaglandin at the inflamed tissue.

Photodermatitis from benzydamine was described in 1980 (14). Cardoso et al (2009) conducted a 4-year retrospective study evaluating the results of photopatch tests (PPTs) performed with an extended series on 83 patients, and among these, 30 were also tested with a non-steroidal anti-inflammatory drug series. Twenty of 30 patients tested (70\%) had a relevant positive PPT to an NSAID, 9 to piroxicam because of systemic photosensitivity, 8 to benzydamine from a topical gel or oral solution, and 2 to ketoprofen (15).

Canelas et al (2010) studied the results of photopatch testing to benzydamine and the clinical features of the dermatitis during a 3-year period (2006-2008). During this period, 74 patients with photodermatoses were photopatch tested with an extended baseline series of allergens including benzydamine and in suspicious cases, with drugs that contain it. Ten patients had a positive photopatch test to benzydamine and to drugs that contain it (Tantum verde oral solution). Nine patients had lower lip cheilitis and one lichenified eczema on photo-exposed sites. The authors concluded that despite its widespread use and its 
known photosensitizing capacity, photoallergic contact dermatitis from benzydamine is probably underdiagnosed as the clinical presentation of mainly the lip and chin is not typical of photoallergic contact dermatitis (16).

On the basis of available data from the literature we decided to include Benzydamine hydrochloride in the list of allergens for photopatch testing. Interestingly, an increased incidence of positive skin patch test reactions after UV-irradiation we observed only to Benzydamine hydrochloride, in the groups of women and in the one of dental professionals. The statistical analysis didn't reveal significant differences, but we could outline dental professionals and women as groups at risk.

Triclosan is a synthetic, broad-spectrum antimicrobial and antifungal agent used extensively for more than 20 years to inhibit bacterial growth on skin and other surfaces. Triclosan was used as a hospital scrub in the 1970s. Now it is prevalent in a variety of consumer products including toothpaste, mouthwash, soaps, shampoos, detergents, bath additives, skin cleansers, deodorants, foot powders and sprays, surgical cleaning treatments, laundry products, disposable paper products, antiodor insoles and hose, as well as in the treatment of textiles and antifungal agent in PVC wetroom carpets (17-19).

Triclosan-containing toothpastes are marginally beneficial in the reduction of tooth cavities. They also reduce dental plaque, gingival inflammation, and gingival bleeding (20). In Norway, Sweden, and Denmark, the main source of exposure is believed to be toothpaste $(17,21,22)$. In Norway, in 2001, toothpaste, for which the upper limit value of triclosan is $0.5 \%$ by weight, accounted for $74 \%$ of the total amount of triclosan applied in personal care products (22).

In the European Union, about $85 \%$ of the total volume of Triclosan is used in personal care products, with the balance going into textiles (5\%) and plastic and food contact materials (10\%) (23). In Europe, Triclosan is regulated as a cosmetic preservative and must be listed on the label (24). On May, 2014, Minnesota governor signed a bill banning the use of Triclosan in most retail consumer hygiene products sold in the state. The ban is set to take effect January 1, 2017 (25).

Triclosan concentrations were associated with allergic sensitization, especially to inhalant and seasonal allergens, rather than to food allergens. Current rhinitis was associated with the highest levels of Triclosan, whereas no association was seen for current asthma. These results are consistent with recent findings in other studies and provide additional evidence for an association between Triclosan and allergy (26). Spanier et al (2014) examined the associations of urinary Triclosan and paraben concentrations with allergic sensitization and asthma in children according to eczema history. They performed a crosssectional analysis of U.S. children aged 6-18 years who participated in the National Health and Nutrition Examination Survey (2005-2006). According to their results, triclosan and paraben exposures may increase the risk of atopic asthma and aeroallergen sensitization (27).

Our results confirm to some extent the concerns about the sensitizing properties of Triclosan. We established the highest rates of contact sensitization to this substance, especially among men (36.7\%) and dental patients (29.4\%), but no reactions of photosensitization. Our results indicate risk of sensitization to Triclosan in consumers, but not in occupational exposure.

Ibuprofen (from the earlier nomenclature iso-butyl-propanoic-phenolic acid) is a non-steroidal antiinflammatory drug (NSAID) originally marketed as Nurofen and since under various trademarks. It is used 
for relief of symptoms of arthritis, primary dysmenorrhoea, fever, and as an analgesic, especially where there is an inflammatory component. Application in dentistry is available as well. It was considered to be photoirritant (28) and photosensitizer (29).

The results achieved from the present pilot study didn't suggest photosensitizing action of Ibuprofen, but relatively high sensitizing potential. On the basis of our results on the sensitization rates we could consider it as the second in importance allergen for all the groups defined by us.

\section{Conclusion}

A pilot study was performed to evaluate the incidence of contact photosensitization to non-steroidal antiinflammatory agents, chemicals used in dental composites and antiseptic agents among dental professionals compared with dental patients. No reactions of photosensitization to Methylhydroquinone and Camphorquinone were observed. The highest rates of contact sensitization to Triclosan, were established, especially among men and dental patients, but no reactions of photosensitization. Our results indicate risk of sensitization to Triclosan in consumers, but not in occupational exposure. We could consider lbuprofen as the second in importance allergen for all the groups defined by us, but no photosensitizing action was found. According to our results, Benzydamine hydrochloride is the only of the substances exerting photosensitizing action, and dental professionals and women could be outlined as groups at risk. However, further work is needed to validate the reliability of these findings.

\section{Acknowledgements}

The study was granted by the Medical University - Sofia, Contract № 48/2014, managed by Prof. Angelina Kisselova, D.D.S., PhD, DSc.

\section{References}

1. Spiewak R. Photoallergies. Post Dermatol Allergol 2009; 26: 347-349.

2. Spiewak R. The Frequency and Causes of Photoallergic Contact Dermatitis among Dermatology Outpatients. Acta Dermatovenerol Croat 2013; 21;4: 230-235.

3. Neumann NJ, Holzle E, Plewig G, et al. Photopatch testing: The 12-year experience of the German, Austrian and Swiss photopatch test group. J Am Acad Dermatol 2000; 42: 183-192.

4. Jindal N, Sharma NL, Mahajan VK, et al. Evaluation of photopatch test allergens for Indian patients of photodermatitis: Preliminary results. Indian Journal of Dermatology, Venereology, and Leprology 2011; 77:2: 148-155.

5. Bruynzeel DP, Ferguson J, Andersen K, et al. Photopatch testing: a consensus methodology for Europe. The European Taskforce for Photopatch Testing. JEADV 2004; 18: 679-682.

6. 6.http://www.chemicalland21.com/specialtychem/perchem/METHYLHYDROQUINONE.htm 
7. Rai R, Dinakar D, Kurian SS, Bindoo YA. Investigation of contact allergy to dental materials by patch testing. Indian Dermatology Online Journal 2014; 5:3: 282-286. doi:10.4103/22295178.137778 .

8. Kamoun EA, Winkelb A, Eisenburgerb M, Menzelaet H. Carboxylated camphorquinone as visiblelight photoinitiator for biomedical application: Synthesis, characterization, and application. Arabian Journal of Chemistry 2014, http://dx.doi.org/10.1016/j.arabjc.2014.03.008.

9. Alvim $\mathrm{HH}$, Alecio AC, Vasconcellos WA, et al. Analysis of camphorquinone in composite resins as a function of shade. Dent. Mater 2007; 23: 1245-1249.

10. Park JY, Chae HK, Rawls HR. Development of a new photoinitiation system for dental light-cure composite resins. Dent Mater 1999; 15: 120-127.

11. Kamoun EA, Menzel H. Crosslinking behavior of dextran modified with hydroxyethyl methacrylate upon irradiation with visible light-effect of concentration, coinitiator type, and solvent. J Appl Polym Sci 2010; 117: 3128-3138.

12. Taira $M$, Urabe $H$, Hirose $T$, et al. Analysis of photo-initiators in visible-light-cured dental composite resins. J Dent Res 1988; 67: 24-28.

13. Santini A, Gallegos IT, Felix CM. Photoinitiators in dentistry: a review. Prim Dent J 2013; 2:4: 3033.

14. Fernandez de Corres L. Photodermatitis from benzydamine. Contact Dermatitis 1980; 6:4: 285.

15. Cardoso J, Canelas MM, Gonçalo M, Figueiredo A. Photopatch testing with an extended series of photoallergens: a 5-year study. Contact Dermatitis 2009; 60: 325-329. doi: 10.1111/j.16000536.2009.01550.x.

16. Canelas MM, Cardoso JC, Gonçalo M, Figueiredo A. Photoallergic contact dermatitis from benzydamine presenting mainly as lip dermatitis. Contact Dermatitis 2010; 63:2: 85-8. doi: 10.1111/j.1600-0536.2010.01707.x.

17. Allmyr M, Adolfsson-Erici M, McLachlan MS, Sandborgh-Englund G. Triclosan in plasma and milk from Swedish nursing mothers and their exposure via personal care products. Sci Total Environ 2006; 372: 87-93.

18. CDC. Fourth national report on human exposure to environmental chemicals. Atlanta: Department of Health and Human Services, Centers for Disease Control and Prevention, 2009.

19. Rodricks JV, Swenberg JA, Borzelleca JF, et al. Triclosan: a critical review of the experimental data and development of margins of safety for consumer products. Crit Rev Toxicol 2010; 40:5: 422-84. doi: 10.3109/ 10408441003667514.

20. Riley $P$, Lamont T. Triclosan/copolymer containing toothpastes for oral health. Cochrane Database of Systematic 2013; 5(12): CD010514. doi: 10.1002/ 14651858. CD010514. pub2. 
21. Borling $\mathrm{P}$, Engelund $\mathrm{B}$, Sørensen $\mathrm{H}$. Kortlægning av triclosan. Kortlægning af kemiske stoffer $\mathrm{i}$ forbrugerprodukter. Nr. 732006. Copenhagen: Danish EPA, 2006.

22. Eide AM. Analyse av triklosan i kosmetiske produkter. Oslo: The Norwegian Food Control Authority, 2003. Available from: http://www.mattilsynet.no/mattilsynet/multimedia/archive/00015/SNT_2003_0_ Analys_15935a.pdf.

23. SCCS. Opinion on triclosan antimicrobial Resistance. 2010; Available from: http://ec.europa.eu/health/scientific_committees/consumer_safety/docs/sccs_o_023.pdf.

24. REGULATION (EC) No 1223 /2009 OF THE EUROPEAN PARLIAMENT AND OF THE COUNCIL of 30 November 2009 on cosmetic products. Official Journal of the European Union. 2009.

25. Minnesota Becomes First State To Ban Antibacterial Chemical Triclosan From Soaps. http://www.huffingtonpost.com/2014/05/20/minnesota-ban-triclosan-antibacterial-soap_ 5357733.html.

26. Bertelsen RJ, Longnecker MP, Løvik M, et al. Triclosan exposure and allergic sensitization in Norwegian children. Allergy 2012; DOI: 10.1111/all.12058.

27. Spanier AJ, Fausnight T, Camacho TF, Braun JM. The associations of triclosan and paraben exposure with allergen sensitization and wheeze in children. Allergy Asthma Proc. 2014; 35(6): 475-81. doi: 10.2500/ aap.2014.35.3803.

28. Galbiati V, Martínez V, Bianchi S, et al. Establishment of an in vitro photoallergy test using NCTC2544 cells and IL-18 production. Toxicol In Vitro 2013; 27:1:: 103-10. doi: 10.1016/j.tiv.2012.09.017.

29. Bergner T, Przybilla B. Photosensitization caused by ibuprofen. Journal of the American Academy of Dermatology 1992; 26:1: 114-116.

\section{Corresponding author}

